\title{
Risikoselektion in der Grundversicherung
}

Christian Baumgartner, André Busato

Korrespondenz: lic. oec. et cand. med. Christian Baumgartner Alte Lützelflühstrasse 26 CH-3415 Hasle-Rüegsau

ctba[at]student.unibe.ch

Prof. Dr. André Busato Institut für Sozial- und Präventivmedizin Finkenhubelweg 11 CH-3012 Bern

abusato[at]ispm.unibe.ch

\section{Einleitung}

Mit der Einführung des Krankenversicherungsgesetzes (KVG) wurde in der Grundversicherung das Prinzip der Kopfprämie verankert, wonach für alle erwachsenen Versicherten einer Krankenkasse dieselbe Prämie gilt (Verbot der Prämiendiskriminierung). Während die Einnahmen pro Versichertem demnach fixiert sind, treten hinsichtlich der Pro-Kopf-Kosten extreme Unterschiede auf. Dieser Umstand vermittelt den Versicherern starke finanzielle Anreize, gesunde Personen aktiv anzuwerben und kranken Personen den Beitritt zu erschweren. Dieses Verhalten wird als Risikoselektion [1] bezeichnet und wurde in der Tagespresse erst kürzlich wieder im Zusammenhang mit der Vergleichsplattform comparis.ch thematisiert [2]. Risikoselektion kann grundsätzlich zur Entstehung grosser, politisch unerwünschter Prämienunterschiede zwischen Gesunden und Kranken führen. Entsprechend stipulierte der Gesetzgeber im KVG einen Aufnahmezwang, der Krankenversicherer verpflichtet, Beitrittswillige vorbehaltlos in die Grundversicherung aufzunehmen [3]. Der Aufnahmezwang soll verhindern, dass Krankenversicherer infolge starker finanzieller Anreize den Beitritt kranker Personen behindern. Inwieweit Krankenversicherer den erwähnten Aufnahmezwang respektieren, wird von staatlicher Seite nicht speziell überwacht. Allerdings würde selbst eine strikte, finanziell aufwendige Durchsetzung des Aufnahmezwanges Versicherer nicht daran hindern, weiterhin Prämiengelder für die Anwerbung Gesunder auszugeben. Es erscheint daher zielführender, die erwähnten, im Kopfprämiensystem prinzipiell immanenten Risikoselektionsanreize zu neutralisieren, bzw. Risikoselektionsgewinne zu eliminieren. Ein erster diesbezüglicher Versuch ist der sogenannte Risikoausgleich. Es handelt sich um einen Umverteilungsmechanismus, in dessen Rahmen Krankenkassen mit jungen, durchschnittlich günstigen Versicherten (gute Risiken) Ausgleichszahlungen an Krankenkassen mit alten, im Durchschnitt teureren Versicherten (schlechte Risiken) leisten. Neben dem Alter werden auch Geschlecht und Wohnort der Versicherten für die Berechnung der Transferzahlungen berücksichtigt.

Unter Gesundheitsökonomen ist derweil unbestritten, dass dieser Risikoausgleich die Effekte der obgenannten Anreize nur unzureichend reduziert [4]: So benötigt z. B. ein vermeintlich «günstiger»

\section{Sélection des risques \\ dans l'assurance de base}

La «chasse aux bons risques» comme on l'appelle aussi est actuellement fréquemment discutée dans les médias. Selon la presse, un comparateur en ligne réputé a contribué à cet engouement en refusant de transférer à certains assureurs (qui avaient payé pour cela) des demandes d'offres de patients. Cette attitude est encouragée par notre système d'assurance à prime unique qui incite les assureurs à attirer les patients en bonne santé et à refuser les malades. Alors qu'il existe de nombreuses publications sur la théorie de la sélection des risques dans l'assurance-maladie, on ne dispose toujours pas de données statistiques exploitables sur le type et l'étendue d'une éventuelle sélection des risques en Suisse. Le but visé par la présente étude consistait donc à récolter des données à ce sujet et à les analyser. Les auteurs de l'étude se sont intéressés d'une part au comportement des assureurs envers les différents groupes d'assurés (par ex. temps de réponse pour les demandes des bons risques et pour celles des mauvais risques) et, d'autre part, aux différences de comportement entre les divers groupes d'assureurs (taux de réponses des grands groupes d'assureurs et des assureurs indépendants). L'analyse des demandes d'offres et des réponses obtenues a mis en lumière des différences significatives tant entre les bons et les mauvais risques qu'entre les différents groupes d'assureurs. Par conséquent, les auteurs recommandent de prendre en compte ces différences lors de la mise en œuvre - si elle se concrétise - du projet de loi tant contesté sur le Managed care.

25-Jähriger aufgrund einer HIV-Infektion eine teure Dauermedikation. Trotzdem muss seine Krankenkasse Beiträge in den Risikoausgleich einzahlen, da 25-Jährige im Durchschnitt relativ gesund sind. Umgekehrt erhält ein Versicherer beispielsweise für eine 
75-jährige Versicherte auch dann Beiträge aus dem Risikoausgleich, wenn die Frau gesund ist. (Daran wird auch der per 1.1.2012 geplante Einbezug von Vorjahresspitalaufenthalten wenig ändern, da ein HIV-Patient bei regelmässiger Medikamenteneinnahme kaum hospitalisiert werden muss.) So erklären die bislang im Risikoausgleich berücksichtigten Faktoren lediglich 20\% der unterschiedlichen individuellen Gesundheitskosten [5]. Ein effektiver Risikoausgleich muss daher den individuellen Gesundheitszustand der Versicherten miteinbeziehen (z.B. via Medikamentengruppen). Dies ist auch im Hinblick auf die kürzlich vom Parlament beschlossene Managed-Care-Vorlage essentiell: Ohne wirksamen Risikoausgleich lohnt es sich für Krankenversicherer bei der Wahl ihrer Managed-Care-Partner, primär Leistungserbringer mit gesundem Patientenstamm zu berücksichtigen. Dies zwingt wiederum die Leistungserbringer, Einfluss auf die Zusammensetzung ihres Patientenstammes zu nehmen bzw. selber Risikoselektion zu betreiben [6]

Zurzeit fehlen öffentlich zugängliche Daten zu Ausmass und Art der Risikoselektion. Diesbezügliche Aussagen haben bislang meist spekulativen Charakter. Das Ziel dieser Studie ist es, anhand realer Offerten statistisch belegbare Erkenntnisse zur Risikoselektion in der obligatorischen Grundversicherung zu gewinnen. Im Mittelpunkt des Interesses stehen dabei Unterschiede im Antwortverhalten der Versicherer gegenüber unterschiedlichen Interessenten.

\section{Methode}

Als private Unternehmen stellen Krankenversicherer aus Sicht Dritter eigentliche Black boxes dar, deren interne Abläufe nicht direkt beobachtbar sind. Ersatzhalber lässt sich die Reaktion der Black box auf standardisierte Anfragen aufzeichnen. Entsprechend wurden alle 47 im Kanton Bern tätigen Krankenversicherer im Oktober und November 2010 mit Offertenanfragen von je 5 «guten Risiken» und 5 «schlechten Risken» beschickt. In den Anfragen wurden ausschliesslich Standard-Grundversicherungen verlangt. Telemedizin- oder Hausarztmodelle wurden explizit ausgeschlossen. Eingehende Offerten wurden in Bezug auf folgende (später erläuterte) Kriterien untersucht:

- Antwortquoten;

- Interne Weiterleitungen innerhalb von Konzernen mit multiplen Tochterkassen (sog. Multikassenkonzerne);

- Antwortzeiten;

- Offerten für Telemedizin-, Hausarzt- oder HMOModelle;

- Offerte von Zusatzversicherungen und Beilage von Gesundheitsfragebogen;

- Vergleich erwarteter und offerierter Prämie

Als gute Risiken wurden 5 Männer im Alter zwischen 23 und 26 Jahren mit Franchisen zwischen 1500 und 2000 Franken für die Studie rekrutiert. Als schlechte Risiken wurden 4 Frauen und 1 Mann im Alter zwischen 72 und 92 Jahren mit Minimalfranchise von 300 Franken eingeschlossen. Die gewünschte Franchisenhöhe dient dabei als Risikosignal. Der Versand wurde so gestaffelt, dass pro Woche und Versicherer höchstens zwei Offertenanfragen eintrafen, da ein gleichzeitiges Eintreffen vieler inhaltlich ähnlicher Anfragen möglicherweise aufgefallen wäre. Um die Entdeckungswahrscheinlichkeit weiter zu reduzieren, wurde das Erscheinungsbild der Briefe und Umschläge für jeden Probanden individuell gestaltet. Der Versand wurde zeitlich so koordiniert, dass Offertenanfragen aus beiden Risikogruppen jeweils am gleichen Tag verschickt wurden, was einen Vergleich der durchschnittlichen Antwortzeiten der beiden Risikogruppen ermöglicht. Die statistische Analyse unterschiedlicher Häufigkeiten erfolgt mit dem FisherTest. Unterschiedliche Mittelwerte (Antwortzeiten) werden mit Hilfe von T-Tests untersucht.

\section{Resultate}

Die Ergebnisse der Befragung sind in Tabelle $1 \mathrm{zu}$ sammengefasst. Als signifikant gelten P-Werte von $\leq 0,05$, sie sind mit einem Sternchen * gekennzeichnet.

$\mathrm{Zu}$ den Punkten 1 bis 3: Die Antwortquote entspricht dem Verhältnis der Anzahl erhaltener Offerten zur Anzahl verschickter Offertanfragen.

$\mathrm{Zu}$ Punkt 4: Als interne Weiterleitungen gelten Fälle, in denen Offertanfragen innerhalb von Multikassenkonzernen zwischen verschiedenen Versicherern weitergegeben werden. Als interne Weiterleitung werden alle Offerten gewertet, die nicht vom angefragten Versicherer, sondern von einem anderen Mitglied desselben Konzerns stammen.

Zu Punkt 5: Als Antwortzeit ist die Anzahl Tage zwischen Versanddatum der Offertanfrage und dem Offerten-Poststempel definiert.

Zu Punkt 6: Anzahl der Fälle, in denen statt- bzw. neben der Grundversicherung unverlangte Zusatzversicherungen offeriert- und Gesundheitsfragebogen beigelegt wurden.

Zu Punkt 7: Vergleich der effektiv offerierten Prämie mit der auf Basis eines finanziell unabhängigen Prämienrechners ermittelten «erwarteten» Prämie. Abweichungen ergeben sich u. a. dann, wenn Versicherer trotz anderslautender schriftlicher Weisung Zusatzversicherungen, Hausarzt-, HMO- oder Telemedizinmodelle offerieren. Weiter treten Abweichungen auf, wenn Offertanfragen innerhalb von Multikassenkonzernen weitergeleitet und von einer anderen als der angefragten Krankenkasse beantwortet werden.

Zu Punkt 8: Vergleich der Gesamtantwortquoten von Multikassenmitgliedern und eigenständigen Krankenkassen. Die Gesamtantwortquote ist definiert als Anzahl aller (z. H. guter und schlechter Risiken) erstellten Offerten im Verhältnis zum Total aller erhaltenen Anfragen. 
Tabelle 1

Ergebnisse der Studie nach 9 verschiedenen Kriterien.

\section{Antwortquote gute vs. schlechte Risiken: alle Versicherer} Anzahl Offertanfragen Anzahl Antworten Anzahl unbeantworteter Anfragen Antwortquote in Prozent
Gute Risiken

224

183

41

81,7 Anzahl Offertanfragen

Anzahl Antworten

Anzahl unbeantworteter Anfragen

Antwortquote in Prozent

3. Antwortquote gute vs. schlechte Risiken:

grösste vier Multikassenkonzerne

Anzahl Offertanfragen

Anzahl Antworten

Anzahl unbeantworteter Anfragen

4. Offertenweiterleitung innerhalb von Multikassenkonzernen

Anzahl Offertanfragen an Multikassenkonzerne

Anzahl interner Weiterleitungen

Anteil interner Weiterleitungen in Prozent

5. Antwortzeit: alle Versicherer

Mediane Antwortzeit in Tagen

Durchschnittliche Antwortzeit in Tagen

6. Zusatzversicherungen und Fragebogen: alle Versicherer

Anzahl Zusatzversicherungen mit Fragebogen

Anzahl Antworten ohne Zusatzversicherung

Zusatzversicherungen mit Fragebogen in Prozent

7. Differenz von erwarteter und offerierter Prämie: alle Versicherer

Unterschreitung der erwarteten Prämie in CHF

8. Gesamtantwortquoten: Multikassen- vs. eigenständige Versicherer Multikassen-Konglomerate

Anzahl erhaltener Offertanfragen

Anzahl erstellter Offerten

Anzahl unbeantworteter Anfragen

Anteil unbeantworteter Anfragen in Prozent

$\begin{array}{ll}\text { sen- vs. eigenständige Versicherer } & \text { M } \\ & 195 \\ & 123 \\ & 72 \\ \text { Pozent } & 36,92 \\ \end{array}$

4,75

173

5,46

18,75
Antwortquote in Prozent

\begin{tabular}{|l|l|}
\hline Schlechte Risiken & P-Wert \\
\hline 218 & \\
\hline 172 & 0,4751 \\
\hline 46 & \\
\hline 78,9 & \\
\hline 93 & \\
\hline 55 & 0,3008 \\
\hline 38 &
\end{tabular}

$82 \quad 75$

$52 \quad 39$

$30 \quad 36$

$\begin{array}{ll}63,4 & 52\end{array}$

0,1953

102

$28 \quad 14$

$24,45 \quad 15,05$

0,0381 *

$4 \quad 4$

4

5,68

0,0001 *

10

2

169

1,17

$0,0367^{*}$

11,05

0,0397 *

\begin{tabular}{|l|l|}
\hline Eigenständige Versicherer & P-Wert \\
\hline 247 & \\
\hline 232 & \\
\hline 15 & $<0,001^{*}$ \\
\hline 6,07 & \\
\hline
\end{tabular}

\section{Grundversicherung vs. Hausarzt- / HMO- / Telemedizinmodelle}

Anzahl Offertanfragen

Anzahl Grundversicherungsofferten

Teuerste 15 Versicherer

64

49

76,56

9

14,06

6

9,38

\begin{tabular}{|c|}
\hline Übrige Versicherer \\
\hline 291 \\
\hline 263 \\
\hline 90,34 \\
\hline 14 \\
\hline 4,83 \\
\hline 14 \\
\hline 4,83 \\
\hline
\end{tabular}

Zu Punkt 9: Deutlich wird, wie oft die 15 teuersten Krankenversicherer trotz anderslautender Anweisung Sparmodelle (Hausarzt-, HMO- oder Telemedizin-Modelle) offeriert haben. Gesondert auf- geführt sind sogenannte Mischofferten, in denen sowohl die verlangte Grundversicherung als auch ein Sparmodell enthalten war. 


\section{Diskussion}

Obwohl die Antwortquoten der schlechten Risiken anreizkonform niedriger ausfallen, ist der betreffende Unterschied statistisch nicht gesichert. Eine ausgedehnte Risikoselektion durch selektives Ignorieren schriftlicher Anfragen erscheint vor diesem Hintergrund unwahrscheinlich. Allerdings fällt auf, dass der erwähnte Trend zulasten der schlechten Risiken bei isolierter Betrachtung der Multikassenkonzerne (Punkt 2) bzw. grössten Multikassenkonzerne (Punkt 3) stärker zutage tritt. Offen bleibt weiter die Frage, ob sich im hypothetischen Falle telefonischer Anfragen (im Gegensatz zur schriftlichen, d. h. belegbaren Form) dasselbe Bild ergäbe.

Betreffend die Gesamtantwortquoten (Punkt 3) fällt auf, dass wirtschaftlich eigenständige Versicherer lediglich 6\%, Mitglieder von Multikassenkonzernen hingegen 36\% aller Anfragen nicht beantworten. Sowohl die niedrigere Gesamtantwortquote als auch der Umstand, dass mehr als jede fünfte Anfrage an Mitglieder von Multikassenkonzernen intern weitergeleitet wird, stützen die These, dass die einzelnen Mitglieder nicht als wirtschaftlich eigenständige Akteure agieren. Die Daten weisen vielmehr darauf hin, dass die Mitglieder sowohl gemeinsames Personal als auch gemeinsame Infrastrukturen nutzen. Hinsichtlich der Antwortzeiten (Punkt 5) findet sich eine statistisch signifikante Differenz zulasten der schlechten Risiken. Dieser Unterschied wird dahingehend interpretiert, dass Anfragen guter Risiken unternehmensintern priorisiert, bzw. die Beantwortung von Anfragen schlechter Risiken verzögert wird. Hierbei erhöht die späte Reaktion des einzelnen Versicherers die Wahrscheinlichkeit, dass der unerwünschte Interessent sich zwischenzeitlich anderweitig versichert. Die Häufung der unverlangten Telemedizin- und HMO-Offerten zuhanden guter Risiken (Punkt 9) deutet darauf hin, dass teure Krankenkassen versuchen, sich mit Hilfe nicht eindeutig bezeichneter Prämiensparmodelle als günstige Versicherer zu präsentieren. Multikassenkonzerne verfügen mit internen Weiterleitungen (zu günstigen Tochterkassen) über ein weiteres Instrument, sich gegenüber guten Risiken günstig zu positionieren (Punkt 4). Entsprechend zeigt sich, dass guten Risiken (im Vergleich zur erwarteten Prämie) trotz Zusatzversicherungen tiefere Prämien offeriert werden als schlechten Risiken (Punkt 7). Bei den erwähnten Zusatzversicherungen handelt es sich ausnahmslos um günstige Zusätze (meist unter 10 Franken pro Monat), mit Hilfe derer sich vor allem Versicherer aus dem preislichen Mittelfeld im Sinne einer Mehrwertstrategie zu differenzieren versuchen. So wurden in erster Linie günstige Auslanddeckungs- bzw. Reiseversicherungszusätze eingeschlossen, von denen ausschliesslich mobile, d.h. durchschnittlich gesündere Individuen profitieren.

\section{Fazit}

Die erhobenen Daten fördern teilweise signifikante Unterschiede im Versichererverhalten gegenüber guten und schlechten Risiken zutage. Dies stützt die eingangs erwähnte These, wonach der aktuelle Risikoausgleich keine effektive Neutralisation der Risikoselektionsanreize erreicht. Hinsichtlich der Multikassenkonzerne lassen gemeinsames Personal, gemeinsame Infrastruktur und mutmasslich freier interner Dokumentenverkehr an der gesetzlich verlangten bzw. nach aussen behaupteten organisatorischen Eigenständigkeit ihrer Tochterkassen zweifeln. Es entsteht hier der Eindruck, dass es sich hierbei in vielen Fällen nicht um Multikassenkonzerne, sondern vielmehr um Multimarkenfirmen handelt, deren einzelne Marken mangels eigener, dezidierter Ressourcen (Personal, Räumlichkeiten, Kapital) keinesfalls eigenständig wirtschaftenden Firmen entsprechen. Letzteres wäre jedoch nach Ansicht der Autoren zwingende Voraussetzung für die Rechtmässigkeit unterschiedlicher Prämien innerhalb eines Multikassenkonzerns. Es fragt sich somit, ob Multikassenstrategien nicht eine grundsätzliche Verletzung des Kopfprämiengebots darstellen. So bleibt zu hoffen, dass die (im Rahmen der Managed-Care-Vorlage) vorgesehene Risikoausgleichsreform in jedem Fall (d.h. auch bei Ablehnung der Gesamtvorlage) umgesetzt wird und die Ära von Risikoselektionsanreizen und Multikassenkonzernen beendet. Sofern die Vorlage indes angenommen wird, sollte die darin vorgesehene Vertragsfreiheit im Managed-Care-Bereich keinesfalls vor der Risikoausgleichsreform in Kraft treten. Kassen hätten ansonsten möglicherweise starke Anreize, Vertragspartner nicht primär nach deren Effizienz, sondern nach der Morbidität ihres Patientenstammes auszuwählen. Zusammenfassend lässt sich sagen, dass es ohne Risikoausgleichsverfeinerung selbst marktfreundlichen Akteuren zunehmend schwerfallen wird, den aktuellen Kassenwettbewerb gegenüber der Idee einer Einheitskasse zu verteidigen.

\section{Literatur}

1 Breyer F et al. Gesundheitsökonomik, 5. überarb. Aufl. Berlin; 2005. S.273ff.

2 Tagesanzeiger. Krankenkassen sortieren Risikokunden aus - und comparis hilft ihnen dabei. Ausgabe vom 27.9.2011.

3 Bundesgesetz über die Krankenversicherung KVG (1994) Art. 4 Abs. 2.

4 Leu RE, Beck C. Risikostrukturausgleich in der Schweiz, Gutachten zuhanden der Technikerkrankenkasse Hamburg. Bern/Luzern; 2006.

5 Spycher S, zit. in: Streit um den Topf der Umverteilung. Der Bund, Ausgabe vom 7.3.2006.

6 Baumgartner C. Aufhebung des Vertragszwangs in der obligatorischen Krankenpflegeversicherung: Voraussetzungen und Umsetzungsvorschläge, Masterarbeit am Volkswirtschaftlichen Institut der Universität Bern; 2007. 